all undergraduates specializing in chemistry. It presents a clear and remarkably concise account. In the space of some twenty pages the terms for symmetry, and state symbols for the description and presentation of spectra, are considered and discussed, as are selection rules and chemical processes. Even such topies as high-pressure, medium-pressure and resonance lamps are covered. These terms are, of course, obvious (or even trivial) to the expert, but often present very real difficulties for the novice and undergraduate.

This chapter is followed by "The Photochemistry of Aromatic Hydrocarbon Solutions" by E. J. Bowen; "Photochemical Gas Phase Reactions in the HydrogenOxygen System" by D. H. Volman; "Photochemistry of Cyclic Ketones" by R. Srinivasan; "Addition of Atoms to Olefins in the Gas Phase" by R. J. Cvetanović; "A New Approach to Mechanistic Organic Photochemistry" by H. E. Zimmerman; "Isotopic Effects and the Mechanism of Energy Transfer in Mercury Photosensitization" by H. E. Gunning and O. P. Strausz; "Photochromism" by R. Dessauer and J. P. Paris; and "Photochemical Rearrangements of Organic Molecules" by O. L. Chapman. These chapters are of a high standard, as is to be expected, for their authors have all made important contributions to various aspects of photochemistry. The range of topics is very wide and it is unlikely that more than a very few will be equally interested in all chapters. This is by no means a disadvantage and could lead to much useful crossfertilization.

Even though the range of subjects in Volume 1 is wide, there are still large areas of photochemistry not mentioned. We are promised that this series will cover topics ranging from the elucidation of spectra of poly. atomic molecules to the photochemistry of biologicel systems in plants and animals. Volume 2, which will follow Volume 1 in quick succession, will doubtless include many of those areas which have not appeared in the first volume. Thereafter, about one volume should appear each yaer.

Advances in Photochemistry is well produced and seems to be remarkably free from misprints, etc. The structural formulæ, especially in the long final chapter, are very clear and beautifully reproduced (which makes a few formulæ in an earlier chapter, which appear to have been drawn by only a semi-skilled hand, look even more out of place). The book can be strongly recommended to all photochemists and will certainly find a place in all science libraries. It is a pity that its price will probably prevent its purchase by a wider audience. The editors, Profs. Noyes, Hammond and Pitts, are to be congratulated on their choice of authors and topics for this first volume, which augurs well for the future of this series.

H. M. Frey

\section{THE SEA AS AN ENVIRONMENT}

\section{Marine Distributions}

Edited by M. J. Dunbar. (The Royal Society of Canada Special Publications, No. 5.) Pp. viii +110. (Toronto: University of Toronto Press; London: Oxford University Press, 1963.) 40s. net.

$\mathrm{T}$

HE publication in book form of a series of papers, the substance of which has been, or probably will be, published in the usual journals, must have behind it a strong belief in the need to direct attention to the interrelationships of the individual subjects. It was the belief that emphasis should be placed on the interdisciplinary nature of oceanography that led to the organization of a symposium in June 1962 at which these six papers on aspects of marine distribution were read. The symposium was to serve, in particular, to introduce the Serial Atlas of the Marine Environment, which was sponsored jointly by the U.S. National Academy of Sciences and the Royal Society of Canada.

The object of the Serial Atlas, which is described in the first paper, is to provide a new type of journal in which distributional investigations from all disciplines will be published. By plotting these distributions on a uniform series of charts prepared by the American Geographical Society (the body responsible for the preparation and publication of the Atlas), it is hoped to build up, over the years, a volume of data in which the separate distributions can be compared. The problem of the comparability of methods, although mentioned, has yet to be resolved.

The second paper is concerned with the vertical distribution of temperature in the upper $100 \mathrm{~m}$ of the eastern Sub-arctic Pacific and describes the processes involved in the development and decline of the seasonal thermocline. This is followed by three papers which directly relate the distribution of physical and chemical properties to biological distribution; they deal with attached algae of the North-east Pacific and zooplankton species in the North Atlantic. One of these papers includes a plea for more taxonomic investigations. It is certainly a feature of the literature that whenever wide-scale distributional investigations are undertaken, many systematic problems have to be cleared up first.

Another paper shows how a knowledge of the life-history of a species increases its value as an indicator of water movements. It might well be added that to understand fully the distribution of a species, a knowledge of its life-history is essential. Such points emphasize the theme of Marine Distributions - that the distribution of plants and animals cannot be examined in isolation. The final paper is concerned with osmoregulation in some of the larger Crustacea, which the author groups according to their capacity to adjust to variations in salinity. This paper, based on experimental work, is refreshingly different from the three previous ones and demonstrates the use of physiological methods in distributional investigations.

The book ends with comments and a summary by Dr. L. A. Walford, without which it would lose a great deal of its value. He links the papers together and raises many of the problems involved in the investigation of the sea as an environment. Although the difficulties he lists are numerous the result is not wholly depressing, as he also suggests ways in which some of them may, at least partially, be overcome. He believes that one approach lies in the "... systematic mapping of all the many elements of the marine environment-currents, bathymetry, bottom sediments, salinity and temperature fields and distributions of all species of plants and animals", which is the plan of the Serial Atlas. Put this way, one may be excused, perhaps, for thinking that this is rather a tall order.

Some comment is necessary on the layout of the papers. Whether, in a publication of this type, abstracts at the heads of the papers are necessary or not, at least a consistent pattern should be followed. Four of these papers have neither abstract nor summary, one has an abstract only and one provides both an abstract and a summary. Some of the figures could be much clearer and it is unfortunate that, in the paper on temperature-salinity-plankton diagrams, Figs. $10 b$ and $11 b$ have been transposed in printing, particularly as it makes the necessary simultaneous comparison of two of the diagrams and their corresponding charts impossible.

Ultimately the success of this book depends on whther it directs attention to relationships between papers that would be overlooked if they were read separately, possibly over a long period. To thoss readers already familiar with the subjects discussed such relationships will probably already be apparent, but to those whose investigations at present appear to have less obvious connexions with these problems this book will be of considerable use.
A. DE C. BAKER 